\title{
Adsorbate and defect effects on electronic and transport properties of gold nanotubes
}

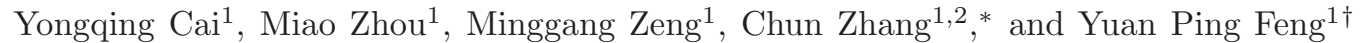 \\ ${ }^{1}$ Department of Physics, National University of Singapore, 2 Science Drive 3, Singapore 117542 \\ ${ }^{2}$ Department of Chemistry, National University of Singapore
}

(Dated: June 26, 2018)

\begin{abstract}
First principles calculations have been performed to study the effects of adsorbates $(\mathrm{CO}$ molecule and $\mathrm{O}$ atom) and defects on electronic structures and transport properties of $\mathrm{Au}$ nanotubes $(\mathrm{Au}(5,3)$ and $\mathrm{Au}(5,5))$. For $\mathrm{CO}$ adsorption, various adsorption sites of $\mathrm{CO}$ on the $\mathrm{Au}$ tubes were considered. Vibrational frequency of the CO molecule was found to be very different for two nearly degenerate stable adsorption configurations of $\mathrm{Au}(5,3)$, implying the possibility of distinguishing these two configurations via measuring the vibrational frequency of $\mathrm{CO}$ in experiment. After $\mathrm{CO}$ adsorption, the conductance of $\mathrm{Au}(5,3)$ decreases by $0.9 G_{0}$, and the conductance of $\mathrm{Au}(5,5)$ decreases by approximately $0.5 G_{0}$. For $\mathrm{O}$ adsorbed Au tubes, $\mathrm{O}$ atoms strongly interact with Au tubes, leading to around $2 G_{0}$ of drop of conductance for both Au tubes. These results may have implications for Au-tube based chemical sensing. When a monovacancy defect is present, we found that for both tubes, the conductance decreases by around $1 G_{0}$. Another type of defect arising from the adhesion of one $\mathrm{Au}$ atom is also considered. For this case, for $\mathrm{Au}(5,3)$ tube, it is found that the defect decreases the conductance by nearly $1 G_{0}$, whereas the decrease of the conductance is only $0.3 G_{0}$ for $\mathrm{Au}(5,5)$ tube due to the adsorption of the extra Au atom.
\end{abstract}

\section{INTRODUCTION}

Gold quasi one-dimensional (1-d) nano structures, such as nanowires and nanotubes, have attracted lots of attention due to their intriguing physical and chemical properties that are very different from bulk gold..$^{1-4}$ The recent rapid progress of experimental techniques enables us to fabricate these gold quasi 1-d structures and measure their novel properties. Long gold monoatom chains have been produced in experiments by depositing single $\mathrm{Au}$ atoms onto a metallic $\mathrm{NiAl}(110)$ surface using a scanning tunneling microscopy(STM) tip,,$\frac{5,6}{,}$ and the unit conductance of such gold chain was reported by another experiment $\underline{7}$ Gold nanowires suspended between two bulk electrodes have been fabricated and intensively studied by either STM techniques 2 or the break junction method $\underline{\underline{3}}$ More recently, helical single-wall gold nanotubes were successfully synthesized in experiment $\underline{4}$ Theoretical studies have shown that among all freestanding gold tubes, $\mathrm{Au}(5,5)$ is the most stable one, and when suspended between two gold electrodes, the Au tube $(5,3)$ is the most energetically favored $\underline{8}$

Owning to their helical structures, gold nanotubes have unique electronic and catalytic properties. It was predicted by theoretical study that the chiral current flowing through gold tubes may induce strong magnetic field $;, 10$ Moreover, both experimental and theoretical investigations have demonstrated excellent catalytic activity of gold nanotubes, 11,12 These unique properties of gold nanotubes suggest promise of gold tubes for future applications of nanoelectronics and nanocatalysis. In this paper, we shall discuss effects of two important

\footnotetext{
*Electronic address: phyzc@nus.edu.sg
}

${ }^{\dagger}$ Electronic address: phyfyp@nus.edu.sg factors that may have great implications for real applications, chemical modifications and defects, on electronic and transport properties of gold tubes.

The chemical modification, in particular, the adsorption or doping of small molecules, has been regarded as a very effective way to tune and control the electronic structure and conductance of quasi 1-d nano structures 13 For instance, for In nanowires, the adatoms of $\mathrm{O}$ and In can individually suppress more than one third of the conductance of the wire $\stackrel{14}{=}$ and the adsorption of a single $\mathrm{CO}$ atom on $\mathrm{Au}$ nanochain turns the system from metal to semiconductor ${ }^{6,15}$ For $\mathrm{Au}$ nanotubes, the interaction of molecules and atoms with the tubes still needs to be clarified. Defects are important because first, in the procedure of fabrication, in principle, defects are inevitable, and second, defects have been found to have significant effects on physical and chemical properties of nano systems, which in many cases are essential for applications, for example, greatly defect-enhanced catalytic activity of gold nano clusters on graphene 16 In the present study, the adsorption of $\mathrm{CO}$ molecule and $\mathrm{O}$ atom, and two types of defects, Au monovacancy and the defect arising from the adsorption of one extra Au atom on the tube, are considered.

\section{COMPUTATIONAL DETAILS}

For structural optimization and electronic structure calculations, the first principles method based on density functional theory (DFT) was employed via the computational package VASP, 17,18 During calculations, a plane wave basis set with the cut-off energy $396 \mathrm{eV}$ was used. The structures were relaxed until the force less than $0.02 \mathrm{eV} / \stackrel{\circ}{A}$. The transport properties were calculated by ATK code within the nonequilibrium Green's function (NEGF) formalism $\stackrel{19-21}{ }$ Double- $\zeta$ polarized basis 

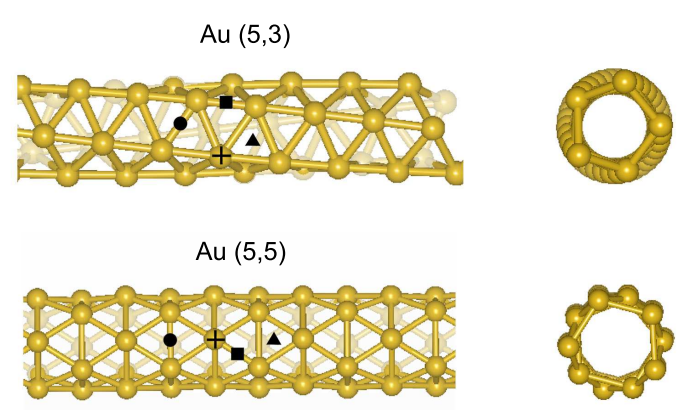

+ : Top

- : B1

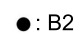

$\Delta$ : Center

FIG. 1: (Color online) Geometric structures of $(5,3)$ and $(5,5)$ Au nanotubes and a schematic description of possible adsorbing sites for adsorption: Top, B1 (Bridge site 1), B2 (Bridge site 2), Center.

and a cutoff energy of $150 \mathrm{Ry}$ for the grid integration were adopted in transport calculations. In all calculations, the Perdew-Burke-Ernzerhof(PBE) format of GGA approximation was included ${ }^{22}$ Vibrational analysis was done using $\mathrm{DMol}_{3}, \stackrel{23,24}{,}$ and the effective core potential (ECP) and a double numerical basis set including a dpolarization function (DND) were adopted.

\section{RESULTS AND DISCUSSION}

\section{A. Adsorption configurations of $\mathrm{CO}, \mathrm{O}$ and $\mathrm{Au}$ on $\mathrm{Au}(5,3) /(5,5)$ tubes}

Fig. 1 shows atomic structures of $\mathrm{Au}(5,3) /(5,5)$ tubes and possible adsorption sites (top, center and two inequivalent bridge sites, B1, B2) of $\mathrm{CO}$ molecule or $\mathrm{O}, \mathrm{Au}$ atoms. In Table I, we list the adsorption energy, bonding length defined as the shortest distance between the adsorbate ( $\mathrm{CO}, \mathrm{O}$ and $\mathrm{Au}$ ) and the nearest $\mathrm{Au}$ atom, and the change of net charge of the adsorbates and the bonded $\mathrm{Au}$ atoms, for most stable adsorption configurations of different cases. In the case of $\mathrm{Au}(5,3)$, for each adsorbate, we found that the difference between the binding energy of the lowest-energy configuration(top site adsorption) and that of the second lowest one(B1 site adsorption) is around $0.03 \mathrm{eV}$. These two adsorption configurations may co-exist in experiments. We therefore show both of them in the table. Other adsorption configurations (not shown in the table) have binding energies at least $0.4 \mathrm{eV}$ lower than the most stable one.

The side views of optimized geometries of $\mathrm{CO}$ or $\mathrm{O}$ adsorbed $\mathrm{Au}(5,3) /(5,5)$ tubes are shown in Fig. 2. For the case of $\mathrm{CO}$ adsorbed $\mathrm{Au}(5,3)$, two nearly degenerate stable adsorption configurations are found as above mentioned: The top site adsorption (Fig.2a) where the C atom directly binds to the $\mathrm{Au}$ atom, and the $\mathrm{B} 1$ adsorp-
TABLE I: Energetics and structures of adsorbates(CO, O, Au) on $\mathrm{Au}(5,3)$ and $\mathrm{Au}(5,5)$ nanotubes. The adsorption energy $\Delta E$ is calculated by subtracting the energy of the total system from the sum of energies of the nanotubes and the adsorbates; $d$ is the shortest length of the bonds formed by $\mathrm{Au}$ and adsorbates; $\delta Q_{x}(x=\mathrm{CO}, \mathrm{O}, \mathrm{Au})$ and $\delta Q_{A u}$ are the net partial charge transfers of the adsorbates and the $\mathrm{Au}$ atom with the shortest distance from the adsorbates.

\begin{tabular}{lcccc}
\hline \hline Adsorbate & $\Delta E(\mathrm{eV})(\mathrm{site})$ & $d(\AA)$ & $\delta Q_{x}$ & $\delta Q_{A u}$ \\
\hline \multirow{2}{*}{$\mathrm{CO}$} & $0.57($ top $)$ & $\mathrm{Au}(5,3)$ & & \\
& $0.54(\mathrm{~B} 1)$ & 2.99 & 0.389 & -0.090 \\
$\mathrm{O}$ & $4.66($ center $)$ & 2.12 & -0.399 & -0.045 \\
& $4.65(\mathrm{~B} 1)$ & 2.03 & -0.528 & 0.150 \\
$\mathrm{Au}$ & $2.28($ center $)$ & 2.73 & -0.060 & 0.170 \\
& $2.27(\mathrm{~B} 2)$ & 2.71 & -0.048 & 0.009 \\
\hline \multirow{2}{*}{$\mathrm{CO}$} & $0.77(\mathrm{~B} 1)$ & $\mathrm{Au}(5,5)$ & & \\
$\mathrm{O}$ & $5.13($ center $)$ & 2.10 & 0.441 & -0.050 \\
$\mathrm{Au}$ & $2.69($ center $)$ & 2.05 & -0.507 & 0.139 \\
\hline \hline
\end{tabular}

tion (Fig.2b) where the $\mathrm{CO}$ is on the bridge site of $\mathrm{Au}$ chains along the direction parallel to the tube axis. For the top site adsorption, the $\mathrm{C}$-Au bonding length is $1.99 \AA$ comparable to that of $\mathrm{CO}$ adsorption on $\mathrm{Au}$ chain $(\mathrm{C}-\mathrm{Au}$

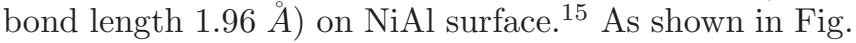
$2 \mathrm{a}$, after the $\mathrm{CO}$ adsorbed on top site, the $\mathrm{Au}(5,3)$ tube undergoes a significant distortion, clearly suggesting the strong interaction between the $\mathrm{CO}$ molecule and $\mathrm{Au}$ tube. For the B1 site adsorption (Fig.2b), the distortion of the tube is much weaker although the adsorption energy in this case is almost the same as the top site adsorption. For the case of $\mathrm{Au}(5,5)$ tube, the lowest energy state occurs when the $\mathrm{CO}$ molecule binds to the B1 site (Fig. 2c). The binding energy, $0.77 \mathrm{eV}$, is higher than the case of $\mathrm{Au}(5,3)$ by about $0.2 \mathrm{eV}$. In all cases, electron transfers from the $\mathrm{CO}$ molecule to the adjacent $\mathrm{Au}$ atom, leading to slightly positively charged CO molecule and negatively charged $\mathrm{Au}$ atom (Table I).

Compared to $\mathrm{CO}$, the binding of $\mathrm{O}$ atom on $\mathrm{Au}$ tubes is much stronger (Table I). For the case of $\mathrm{Au}(5,3)$, similarly, there are two nearly degenerate adsorption configurations, the center (Fig. 2d) and the B1 site (Fig. 2e) adsorption, with the adsorption energy 4.66 and $4.65 \mathrm{eV}$ respectively. For $\mathrm{Au}(5,5)$, the center site adsorption (Fig. $2 \mathrm{f}$ ) is the most stable configuration with the adsorption energy of $5.13 \mathrm{eV}$. When binded on B1 site of $\mathrm{Au}(5,3)$, a significant distortion of the $\mathrm{Au}$ tube occurs. Moreover, a relatively larger electronegativity of $\mathrm{O}$ atom enables a net partial charge transfer from $\mathrm{Au}$ to the adsorbate and makes gold atoms positively charged (Table I).

The adsorption of one extra $\mathrm{Au}$ atom on $\mathrm{Au}$ tubes is also considered. For both $\mathrm{Au}$ tubes, the adsorption energy of the lowest energy configuration is larger than 2.2 $\mathrm{eV}$ (Table I), suggesting that in the procedure of fabrication, it is very possible to have Au adatoms on Au tubes. Later, we will show how the Au adatom affects electronic and transport properties of $\mathrm{Au}$ tubes. 
(a)

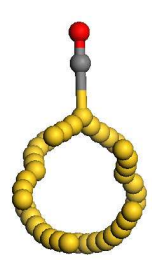

(d)

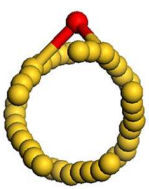

(b)

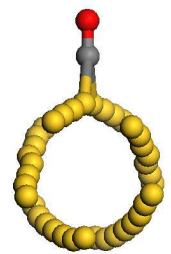

(e)

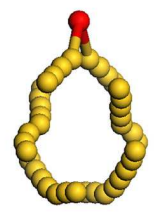

(c)

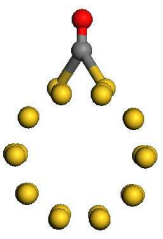

(f)

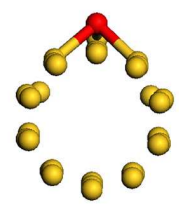

FIG. 2: (Color online) Adsorption geometries for CO adsorption on $\mathrm{Au}(5,3)$ at top (a), B1 (b), and $\mathrm{Au}(5,5)$ at B1 (c) sites, oxygen adsorption on $\mathrm{Au}(5,3)$ at center (d), B1 (e), and $\mathrm{Au}(5,5)$ at center (f) sites, respectively. The $\mathrm{C}, \mathrm{O}$, and $\mathrm{Au}$ atoms are depicted in grey, red, and yellow, respectively.

\section{B. Electronic structures of $\mathrm{CO}$ and $\mathrm{O}$ adsorbed $\operatorname{Au}(5,3) /(5,5)$ tubes}

Here, we describe the electronic structures of $\mathrm{CO} / \mathrm{O}$ absorbed $\mathrm{Au}(5,3)$ and $\mathrm{Au}(5,5)$ tubes. First, to understand the interaction between $\mathrm{CO}$ molecule and $\mathrm{Au}$ tubes, we plot in Fig. 3 local density of states (LDOS) projected on the $\mathrm{CO}$ molecule and the binded $\mathrm{Au}$ atom. Similar to the case of $\mathrm{CO}$ binding to Au nano clusters, 25 the highest occupied molecular orbital(HOMO) and the lowest unoccupied molecular orbital(LUMO) of CO have the biggest contribution to the chemisorption since the energy levels of these two states change significantly upon adsorption due to the hybridization with Au states. For top site adsorption on $\mathrm{Au}(5,3)$ (Fig. 3a), the CO HOMO orbital $5 \sigma$ is pushed below the $1 \pi$ orbital whose energy level doesn't change much, and the LUMO orbital $2 \pi^{*}$ is pushed below Fermi energy and overlap with the whole continuous $d$ band of Au, leading to the partial population of this orbital (the backdonation process ${ }^{26,27}$ ). The amount of electrons backdonated to the $2 \pi^{*}$ orbital can be roughly estimated by integrating the LDOS of CO within the energy range of the $\mathrm{Au} d$ band. In this case, about 0.9 electron is transferred from $\mathrm{Au} d$ bands to the CO anti-binding orbital $2 \pi^{*}$. In the figure, we also plot the wave functions of several molecular orbitals of $\mathrm{CO}$

after adsorption, from which we can see that indeed the $5 \sigma$ orbital strongly hybridize with Au states, and $4 \sigma$ as well as two degenerate $1 \pi$ orbital $(1 \pi-1$ and $1 \pi-2)$ only weakly interact with the Au tube. When $\mathrm{CO}$ adsorbs on B1 site of $\mathrm{Au}(5,3)$ (Fig. 3b), differently from top site adsorption, $1 \pi$ orbital also contributes to the chemisorption by significantly hybridizing with Au states, and as a result, the degeneracy between $1 \pi-1$ and $1 \pi-2$ is lifted. Amount of electrons backdonated to $2 \pi^{*}$ orbital in this case (1.57 electrons) is estimated to be much larger than that of the top site adsorption.

The significantly different backdonation strength between the top site and B1 site adsorption implies a way to distinguish these two almost degenerate adsorption configurations of $\mathrm{CO}$ on $\mathrm{Au}(5,3)$ in experiment. The more the $\mathrm{CO}$ anti-binding $2 \pi^{*}$ orbital is backdonated, the weaker the $\mathrm{CO}$ bond will be. The strength of the $\mathrm{CO}$ bond can be seen from the bond length and also the vibrational frequency of the stretching mode of the molecule, the latter of which can be measured in experiment. Indeed, the CO bond length of the B1 adsorption, $1.165 \AA$, is larger than that of the top adsorption, 1.151 $\AA$ due to the stronger backdonation. Note that the bond length of free $\mathrm{CO}$ molecule is $1.128 \AA$ (Our calculated value of $1.146 \AA$ ). The vibrational frequency of the CO stretching mode is calculated to be $2094 \mathrm{~cm}^{-1}$ for top adsorption and $1919 \mathrm{~cm}^{-1}$ for B1 adsorption. The difference between these two vibrational frequencies should be able to be seen in experiment. As a test of the method of calculating vibrational spectra, for the same mode of the free $\mathrm{CO}$ molecule, our calculations give the frequency of $2128 \mathrm{~cm}^{-1}$, which is in good agreement with the experimental value of $2140 \mathrm{~cm}^{-1}, 28$

The backdonation of $2 \pi^{*}$ orbital, and the splitting of $1 \pi-1$ and $1 \pi-2$, are also observed for the lowest energy adsorption of $\mathrm{CO}$ on $\mathrm{Au}(5,5)$, the $\mathrm{B} 1$ adsorption (Fig. $3 \mathrm{c})$. Here, the nearly $1 \mathrm{eV}$ of the splitting of two $1 \pi$ orbitals is much larger than that of the B1 adsorption of $\mathrm{Au}(5,3)$ (about $0.5 \mathrm{eV}$ ). The $\mathrm{CO}$ bond length in this case is calculated to be $1.172 \AA$, and the corresponding vibrational frequency of the $\mathrm{CO}$ stretching mode is 1922 $\mathrm{cm}^{-1}$, about the same as that of the B1 adsorption on $\mathrm{Au}(5,3)$.

In Fig. 4, we show the LDOS of the Au atom binded with the $\mathrm{O}$ before and after $\mathrm{O}$ adsorption. Again, for $\mathrm{Au}(5,3)$, there are two nearly degenerate adsorption configurations, the center and the B1 adsorption, and for $\mathrm{Au}(5,5)$, the most stable configuration occurs when the $\mathrm{O}$ atom binds to the center position. For all cases, the $\mathrm{O}$ adsorption causes great changes of LDOS of the binded $\mathrm{Au}$ atom, indicating the strong interaction between the $\mathrm{O}$ and the Au tube.

\section{Conductance of $\mathrm{CO} / \mathrm{O}$ absorbed $\mathrm{Au}$ tubes}

Now, we turn to the analysis of conductance of chemically modified Au tubes. It has been reported that for 
(a)

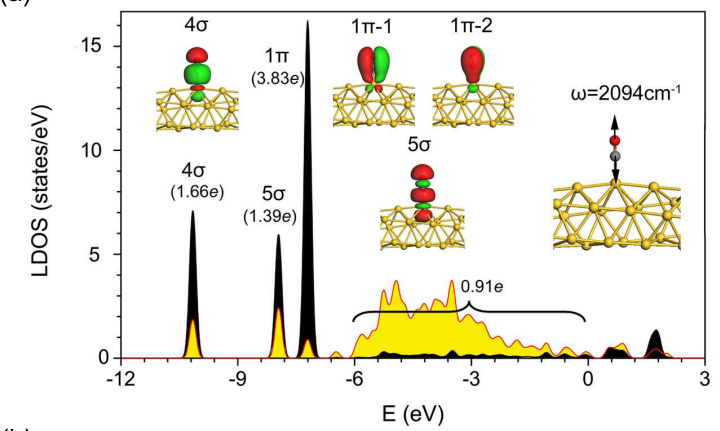

(b)

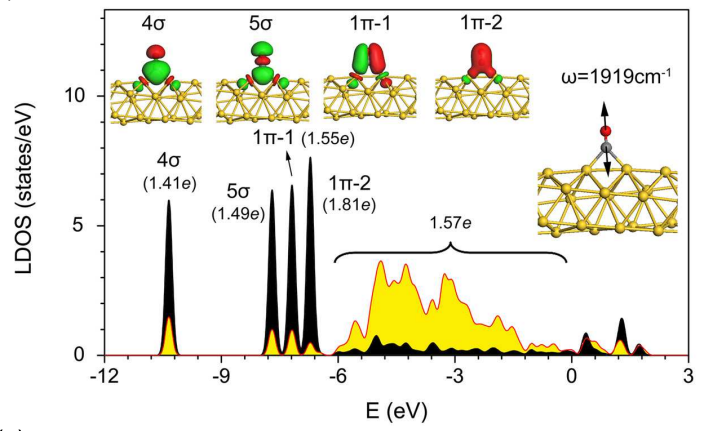

(c)

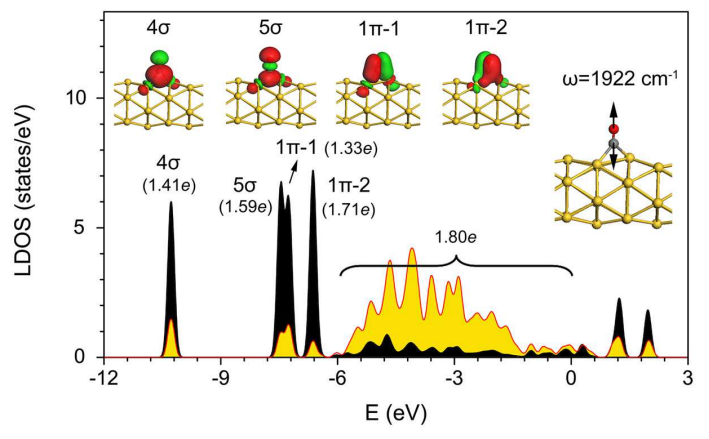

FIG. 3: (Color online) LDOS and orbitals of CO adsorbed on $\mathrm{Au}(5,3)$ at top(a), $\mathrm{B} 1(\mathrm{~b})$ sites and $\mathrm{Au}(5,5)$ at $\mathrm{B} 1(\mathrm{c})$ site. The LDOS projected on the $\mathrm{CO}$ molecule is shown in black and that projected on the gold atom with the shortest distance from the $\mathrm{C}$ atom is colored yellow. The populations of $\mathrm{CO}$ orbitals are calculated through integrating over the relevant peaks. Included also are the vibrational frequencies of stretching mode for different adsorption configurations. The softening frequencies are evidence of the back-donation process during adsorption. Notice that the frequency of free $\mathrm{CO}$ molecule is calculated as $2128 \mathrm{~cm}^{-1}$

an Au monoatom chain that has one unit of conductance, the adsorption of a single CO molecule can make a sharp modification of conductance of Au monochain from $1 G_{0}$ to 0 after adsorption at top site. 15 It would be interesting to see how the adsorption of $\mathrm{CO}$ or $\mathrm{O}$ modify the conductance of $\mathrm{Au}(5,3)$ or $(5,5)$ tubes that essentially are the aggregation of five strands of Au atoms.

The calculated conductance spectra of the pristine and $\mathrm{CO}$ adsorbed $\mathrm{Au}(5,3)$ and $\mathrm{Au}(5,5)$ are shown in Fig. 5. The conductance of both pristine tubes at Fermi en- (a)

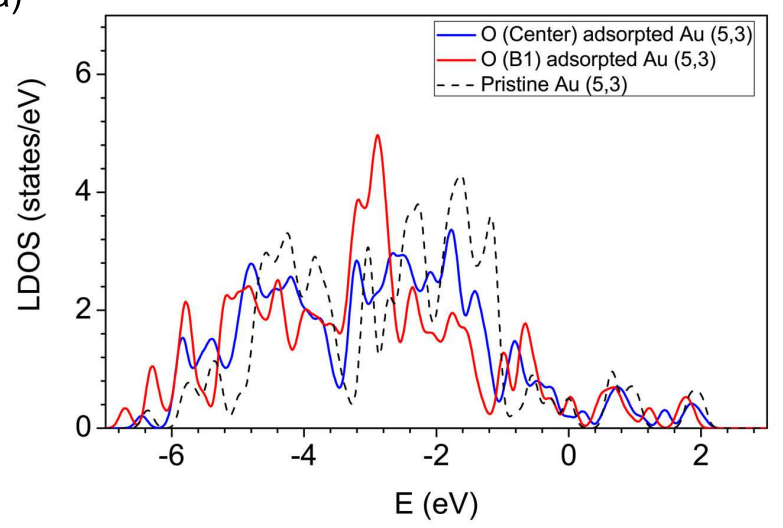

(b)

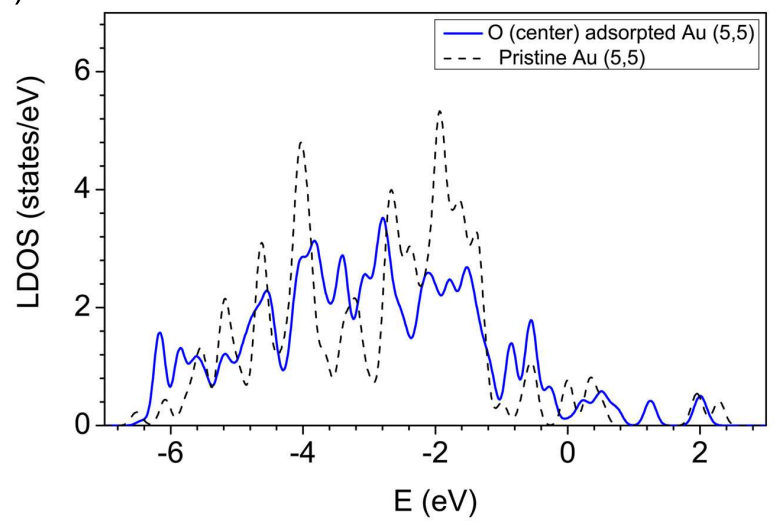

FIG. 4: (Color online) Comparison of Au states after $\mathrm{O}$ atomic adhesion with those of the pristine $(5,3)(\mathrm{a})$ and $(5,5)$ (b) $\mathrm{Au}$ tubes. For the adsorbed tubes, LDOS is projected onto $\mathrm{Au}$ atom with the shortest distance from the $\mathrm{O}$ atom.

ergy is $5 G_{0}$, in agreement with previous studies $\stackrel{8}{=}$ For $\mathrm{Au}(5,3)$, the $\mathrm{CO}$ adsorption decreases the conductance at Fermi energy of the tube by $0.9 G_{0}$ for both top and $\mathrm{B} 1$ adsorptions, suggesting that $\mathrm{Au}(5,3)$ may be used as a chemical sensor for $\mathrm{CO}$ molecules. For $\mathrm{Au}(5,5)$, the lowest-energy configuration of $\mathrm{CO}$ adsorption decreases the conductance at Fermi energy of the tube by $0.5 G_{0}$. Compared to $\mathrm{CO}$, effects of $\mathrm{O}$ atom on transport properties are much more pronounced due to the stronger interaction between the $\mathrm{O}$ atom and the $\mathrm{Au}$ tube (Fig. 6 ). For both tubes, the O adsorption causes the drop of the conductance at Fermi energy by around $2 G_{0}$.

\section{Defects effect on conductance of Au tubes}

Finally, we discuss the effects of two types of defects, the Au monovacancy and the defect arising from the adhesion of one extra Au atom, on conductance of Au tubes. In Table I, we give the adsorption energies of a single $\mathrm{Au}$ atom on $\mathrm{Au}(5,3)(2.28 \mathrm{eV} @$ center and $2.27 \mathrm{eV} @ \mathrm{~B} 2)$ and $\mathrm{Au}(5,5)$ (2.69eV@center). The formation energies of a 


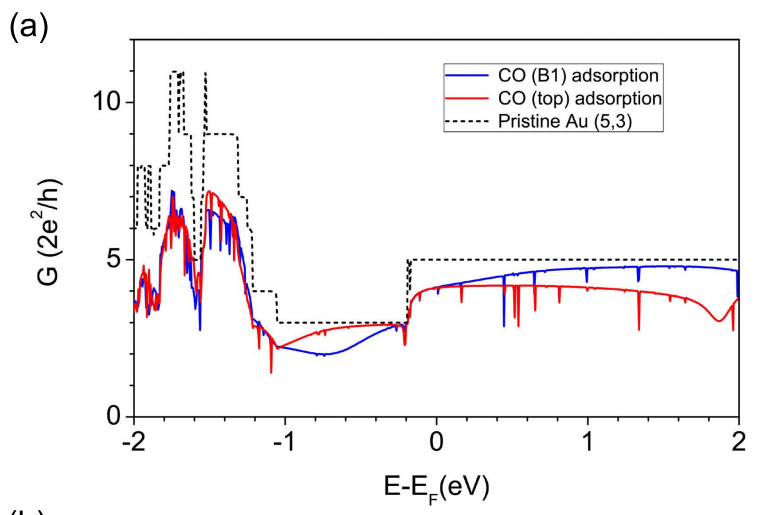

(b)

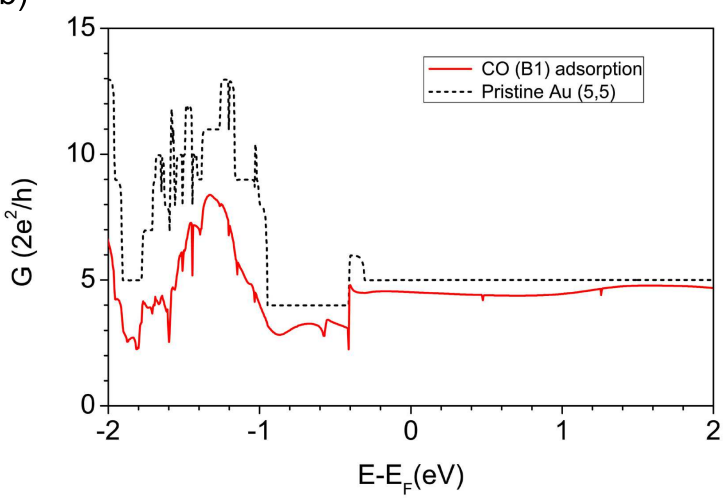

FIG. 5: (Color online) Quantum conductance spectrum for $\mathrm{CO}$ adsorbed $\mathrm{Au}(5,3)(\mathrm{a})$ and $\mathrm{Au}(5,5)(\mathrm{b})$. The conductance spectra of pristine tubes(dashed lines) are also given for comparison

monovacancy are calculated to be 3.32 and $3.52 \mathrm{eV}$ for $\mathrm{Au}(5,3)$ and $\mathrm{Au}(5,5)$ respectively, which are rather small compared to those of CNTs $\frac{29}{2}$ The high adsorption energies of one $\mathrm{Au}$ atom and relatively low formation energies of monovancy suggest that these two types of defects are likely to occur in the fabrication of Au tubes.

The conductance spectra and optimized structures of defective $\mathrm{Au}(5,3)$ are given in Fig. 7. For cases of the extra $\mathrm{Au}$ atom adsorbed on the tube (B2 and center sites), after relaxation, pyramid-like structures are formed as shown in Fig. 7a. The conductance spectra of both two adsorption configurations show very similar behaviors, and at Fermi energy, the conductance decreases by about $0.8 G_{0}$ for both configurations. For Au monovacancy, the defect decreases the conductance of the tube at Fermi energy about $1.2 G_{0}$ (Fig. 7b). Similarly, the Au monovacancy on $\mathrm{Au}(5,5)$ causes a $0.9 G_{0}$ of drop of conductance at Fermi energy (Fig. 8). The Au adatom on $\mathrm{Au}(5,5)$ only leads to a decrease of $0.3 G_{0}$ of the tube as we can see from Fig. 8. (a)

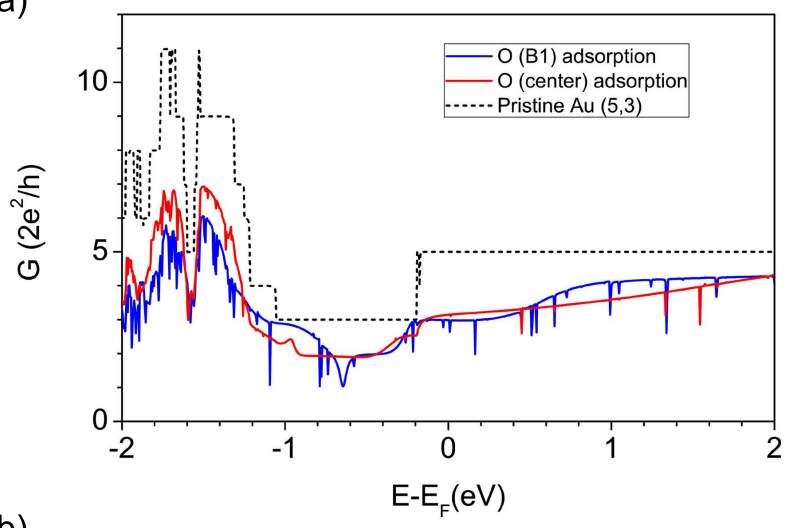

(b)

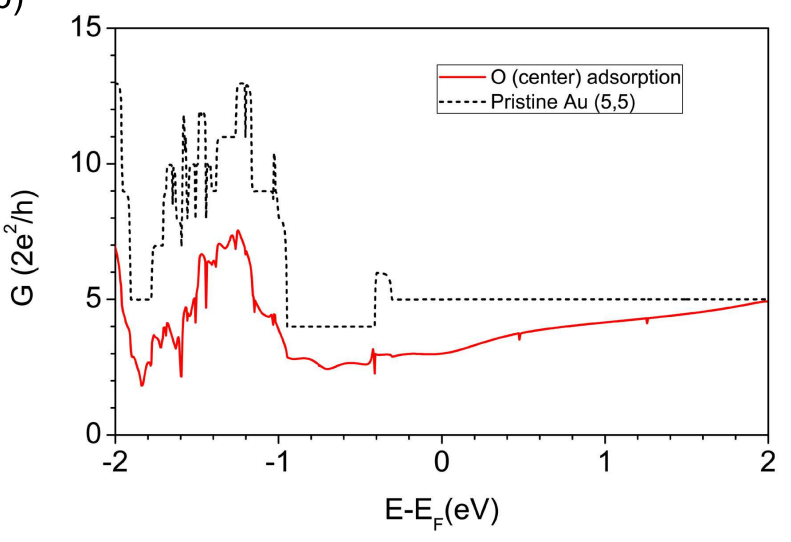

FIG. 6: (Color online) Quantum conductance spectrum for oxygen adsorbed $\mathrm{Au}(5,3)(\mathrm{a})$ and $\mathrm{Au}(5,5)(\mathrm{b})$. The conductance spectra of pristine tubes(dashed lines) are also given for comparison

\section{CONCLUSIONS}

We have investigated via the first principles method based on DFT the influence of adsorbates (CO molecule and $\mathrm{O}$ atom) and defects on the the electronic and transport properties of $\mathrm{Au}(5,3)$ and $\mathrm{Au}(5,5)$ nanotubes. For $\mathrm{Au}(5,3)$, we found that there are two nearly degenerate stable adsorption configurations(top site and B1 site adsorption) for both $\mathrm{CO}$ molecule and $\mathrm{O}$ atom. For the case of CO, for both tubes, HOMO and LUMO states of $\mathrm{CO}$ contribute the most to the chemisorption. For one of these two degenerate configurations of $\mathrm{Au}(5,3)$, the $\mathrm{B} 1$ site adsorption, and the stable adsorption configuration of $\mathrm{Au}(5,5)$, the $1 \pi$ orbital is also involved in the bonding between $\mathrm{CO}$ and $\mathrm{Au}$ tube. The charge transfer between the $\mathrm{CO}$ molecule and both Au tubes is dominated by socalled backdonation process in which the electrons transferred from $\mathrm{Au} d$ bands to the $\mathrm{CO}$ anti-binding $2 \pi^{*}$ orbital. We also predicted a significant difference between the vibrational frequencies of the $\mathrm{CO}$ molecule for two degenerate stable adsorption configurations of $\mathrm{Au}(5,3)$, which may be used to distinguish these two configurations in experiments. 
(a)

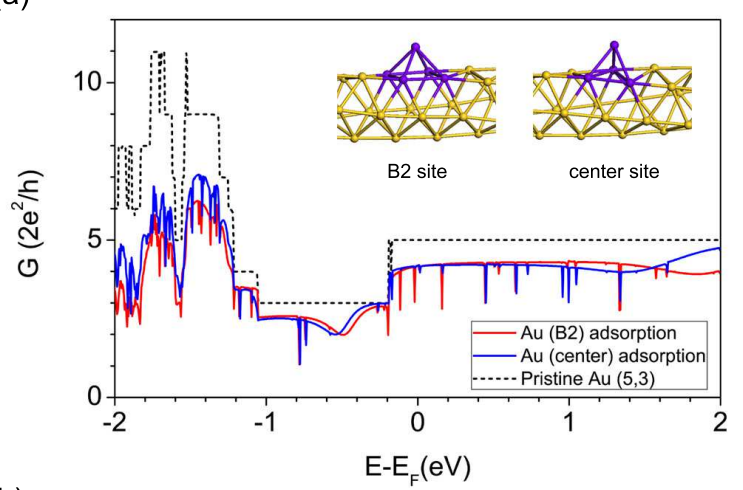

(b)

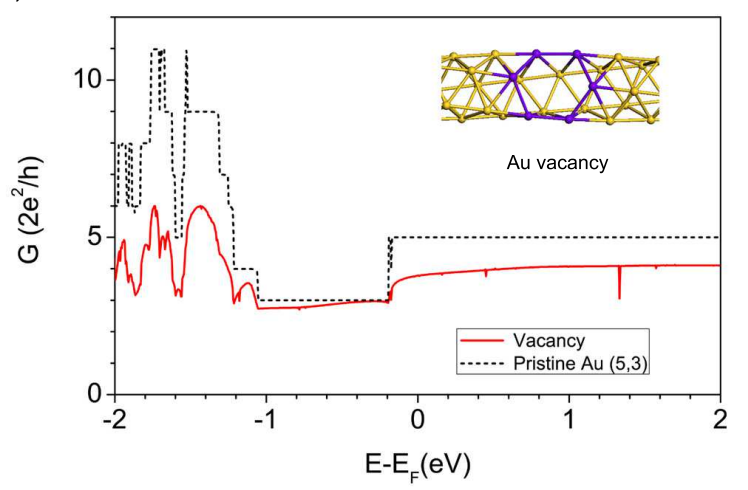

FIG. 7: (Color online) Conductance as a function of energy for defective $\mathrm{Au}(5,3)$ tube with defects arising from $\mathrm{Au}$ adhesion(a) and monovacancy(b) on the tube. The relaxed defective structures are given as the insets, where the atoms around the defective site are highlighted by violet balls. It can be seen that the Au adatom distorts the tube differently at different adsorption sites.

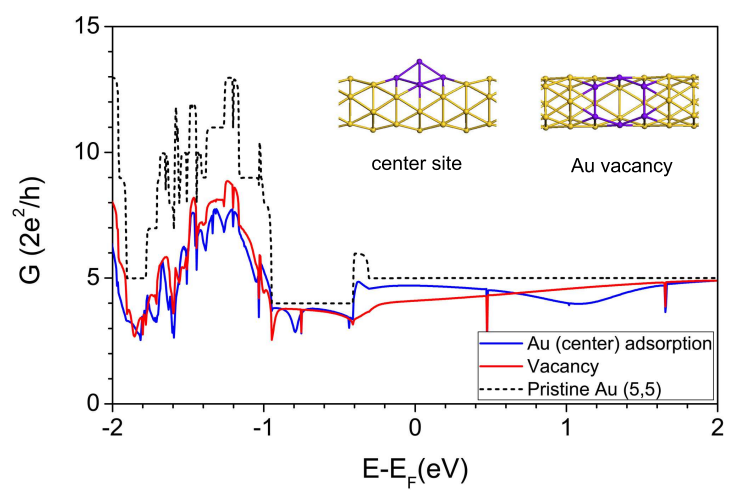

FIG. 8: (Color online) Conductance as a function of energy for defective $\mathrm{Au}(5,5)$ tube with defects arising from $\mathrm{Au}$ adhesion(blue line) and monovacancy(red line) on the tube. The relaxed defective structures are given as the insets, where the atoms around the defective site are highlighted by violet balls.

${ }^{1}$ Y. Kondo and K. Takayanagi, Science 289, 606 (2000).
After $\mathrm{CO}$ adsorption, the conductance of $\mathrm{Au}(5,3)$ decreases by $0.9 G_{0}$, and the conductance of $\mathrm{Au}(5,5)$ decreases by approximately $0.5 G_{0}$. For $\mathrm{O}$ adsorbed $\mathrm{Au}$ tubes, $\mathrm{O}$ atoms strongly interact with $\mathrm{Au}$ tubes, leading to around $2 G_{0}$ of drop of conductance for both $\mathrm{Au}$ tubes. These results may have implications for Au-tube based chemical sensing. When a monovacancy defect is present, we found that for both tubes, the conductance decreases by around $1 G_{0}$. Another type of defect arising from the adhesion of one Au atom is also considered. For this case, it is found that the defect decreases the conductance by nearly $1 G_{0}$ for $\mathrm{Au}(5,3)$ tube, and the decrease of the conductance is around $0.3 G_{0}$ for $\mathrm{Au}(5,5)$ tube.

Acknowledgement: This work was supported by NUS Academic Research Fund (Grant Nos: R-144-000-237133 and R-144-000-255-112). Computations were performed at the Centre for Computational Science and Engineering at NUS.

${ }^{2}$ H. Ohnishi, Y. Kondo, and K. Takayanagi, Nature 395, 
780 (1998).

3 N. Agraït, A. L. Yeyati, and J. M. van Ruitenbeek, Phys. Rep. 377, 81 (2003).

4 Y. Oshima, A. Onga, and K. Takayanagi, Phys. Rev. Lett. 91, 205503 (2003).

5 N. Nilius, T.M. Wallis, and W. Ho, Science 297, 1853 (2002).

6 N. Nilius, T.M. Wallis, and W. Ho, Phys. Rev. Lett. 90, 186102 (2003).

7 A. I. Yanson, G. Rubio Bollinger, H. E. van den Brom, N. Agrait, and J. M. van Ruitenbeek, Nature 395, 783 (1998)

8 R. T. Senger, S. Dag, and S. Ciraci, Phys. Rev. Lett. 93, 196807 (2004).

9 T. Ono, and K. Hirose, Phys. Rev. Lett. 94, 206806 (2005).

10 D. Z. Manrique, J. Cserti, and C. J. Lambert, Phys. Rev. B 81, 073103 (2010).

11 M. A. Sanchez-Castillo, C. Couto, W. B. Kim, and J. A. Dumesic, Angew. Chem. Int. Ed. 43, 1140 (2004).

12 W. An, Y. Pei, and X. C. Zeng, Nano Lett. 8, 195 (2008).

13 C. Zhang, R. N. Barnett, U. Landman, Phys. Rev. Lett. 100, 046801 (2008).

14 S. Wippermann, N. Koch, and W. G. Schmidt, Phys. Rev. Lett. 100, 106802 (2008)

15 A. Calzolari, C. Cavazzoni, and M. B. Nardelli, Phys. Rev. Lett. 93, 096404 (2004).

16 M. Zhou, A. Zhang, Z. Dai, C. Zhang, and Y. P. Feng, J.
Chem. Phys. 132, 194704 (2010)

17 G. Kresse, Furthmller, J. Comput. Mater. Sci. 6, 15 (1996).

18 G. Kresse, Furthmller, Phys. Rev. B 54, 11169 (1996).

19 M. Brandbyge, J. -L. Mozos, P. Ordejn, J. Taylor, K. Stokbro, Phys. Rev. B 65, 165401 (2002).

20 J. M. Soler, E. Artacho, J. D. Gale, A. Garca, J. Junquera, P. Ordejn, D. Snchez-Portal, J. Phys. Condens. Matter 14, 2745 (2002).

21 J. Taylor, H. Guo, J. Wang, Phys. Rev. B 63, 245407 (2001).

22 J. Perdew, K. Burke, M. Ernzerhof, Phys. Rev. Lett. 77, 3865 (1996).

23 B. Delley, J. Chem. Phys. 92, 508 (1990).

24 B. Delley, J. Chem. Phys. 113, 7756 (2000).

25 B. Yoon, H. Häkkinen, U. Landman, A. S. Wörz, J.-M. Antonietti, S. Abbet, K. Judai, U. Heiz, science 307, 403 (2005).

26 G. Blyholder, J. Phys. Chem. 68, 2772 (1964).

27 M. Kiguchi, D. Djukic, and J. M. van Ruitenbeek, Nanotechnology 18, 035205 (2007).

28 H.-J. Freund and G. Pacchioni, Chem. Soc. Rev. 37, 2224(2008).

29 B. Biel, F. J. García-Vidal, Á. Rubio, and F. Flores, J. Phys. Condens. Matter, 20, 294214 (2008). 